This is the accepted manuscript of the article, which has been published in Proceedings of the 21st International Academic Mindtrek Conference. New York, NY: ACM. ISBN: 978-1-4503-5426-4. http://dx.doi.org/10.1145/3131085.3131088

\title{
Field-of-View Extension for VR Viewers
}

\author{
Ismo Rakkolainen, Roope Raisamo \\ University of Tampere \\ Faculty of Communication Sciences \\ 33014 Tampere, Finland \\ $+358-50-3185894$ \\ \{ismo.rakkolainen | roope.raisamo\}@uta.fi
}

Poster

\author{
Matthew Turk, Tobias Höllerer \\ University of California \\ Department of Computer Science \\ Santa Barbara, CA 93106, USA \\ $+1-805-893-4321$ \\ \{mturk| holl\}@cs.ucsb.edu
}

\begin{abstract}
We present a prototype of a smartphone-based virtual reality (VR) viewer, which can cover nearly the full human field-of-view (FOV). The prototype suggests that such extensions are feasible ways to significantly expand the FOV of standard VR viewers. The concept can be employed for future VR viewers or it can even be retrofitted to some existing ones.
\end{abstract}

\section{Categories and Subject Descriptors}

I.3.7 [Computer graphics]: Three-Dimensional graphics and realismvirtual reality; H.5.2 [Information interfaces and presentation]: User interfaces-input devices and strategies. I.4.0 [Image processing and computer vision]: General — image displays; H.5.1 [Information interfaces and presentation (e.g., HCI)]: Multimedia information systems — artificial, augmented, and virtual realities

\section{Keywords}

VR viewers; field-of-view; virtual reality; $360^{\circ}$ video.

\section{INTRODUCTION}

Head-mounted displays (HMD) and virtual reality (VR) viewers are often used to view VR and cinematic, spherical $360^{\circ}$ (horizontal) $\times 180^{\circ}$ (vertical) video $\left(360^{\circ}\right.$ video $)$. VR has recently become feasible and affordable for consumers in the form of low-cost, smartphone-based VR viewers, which use an inserted smartphone as a display. A wide field-of-view (FOV) can improve the sense of immersion and performance in some tasks in virtual environments. Wide FOV is rare in HMDs and VR viewers.

Our contribution in this paper is to demonstrate a super-wide FOV extension for a VR viewer. We used a Google Cardboard as an example, but similar concepts can be designed or retrofitted to other VR viewers and HMDs. We first discuss wide-FOV issues and previous HMDs, then present our prototype, discuss its limitations and potential improvements, and finally give conclusions.

\footnotetext{
${ }^{1} \mathrm{http}: / /$ www.fakespacelabs.com/Wide5.html

${ }^{2} \mathrm{http}: / /$ www.starvr.com/

${ }^{3}$ http://vrunion.com/
}

\section{PREVIOUS WIDE-FOV HMDS}

The precision area of human vision (foveal vision) is just a few degrees wide, but due to eye movement and saccades, humans perceive as if it were larger. Peripheral vision on the extreme sides has very low precision. Beyond $50^{\circ}$ most humans cannot see details or read text. Most of the time humans direct gaze towards the front, not to the low-precision extreme sides (peripheral vision) [18]. Also binocular and color vision, light sensitivity and the ability to perceive shape and motion vary across the visual field.

Wide-FOV displays convey peripheral information, improve orientation, situational awareness, object avoidance and performance in some tasks and are generally preferred by audiences. They also have an impact on perceptional issues such as distance judgment, motion sickness and others [2], [12], [15], [17], [21], [22]. Even though wide FOV is generally conducive to simulator sickness, the wide FOV itself is not a problem, as the natural human FOV attests, but rather the insufficient implementation of HMDs and conflicts of various sensory stimuli.

Many requirements and parameters for an HMD need to be balanced [8]; e.g., wide FOV and high resolution are contradictory goals, as a wide FOV distributes the available pixels over a wider angle, reducing their spatial resolution. Other HMD parameters are exit pupil size, latency, weight, price etc.

Sutherland implemented the first HMD employing 3D graphics and head tracking in 1968 [23]. Since then numerous HMDs have been designed [6], [14], [5].

LEEP optics [10] was the first to deliver feasible wide FOV for HMDs. Kiyokawa [13], FakeSpace Wide5 ${ }^{1}$, StarVR ${ }^{2}$, VRUnion Claire $12 \mathrm{M}^{3}$ and some other prototype HMDs have wide FOV, e.g., Luckey built a $270^{\circ}$ FOV HMD ${ }^{4}$. Rakkolainen et al. [20] has presented a super-wide optical design, which curves both lenses and screen seamlessly around the eyes. Tiled displays merge multiple lenses and micro-displays seamlessly to increase FOV without reducing resolution [4], [7]. Also peripheral lights without any image have been proposed [3], [12], [24].

Other approaches to mitigate the problems caused by the narrow FOV of HMDs are to compress a wide image to fit it into the FOV of an HMD [1], [16] or to augment parts of it to the natural FOV [9], or to amplify the head movement [11].

Currently popular smartphone-based VR viewers have limited FOV (e.g., Google Cardboard $\sim 65^{\circ} \times 65^{\circ}$, Samsung Gear VR $101^{\circ} \times 101^{\circ}$ ). Wearality Inc. employs custom Fresnel lenses for their

\footnotetext{
${ }^{4} \mathrm{http}: / /$ www.roadtovr.com/oculus-rift-creator-built-120-and-270-degreefov-hmd-prototypes/
} 
wide-FOV $\left(150^{\circ} \times 123^{\circ}\right)$ VR viewers ${ }^{5}$. The natural human FOV is still much wider $\left(\sim 210^{\circ} \times 135^{\circ}\right)$.

Humans are accustomed to live and act with a natural wide FOV, and it is also required for many tasks. If a person had as limited a FOV as of a typical VR viewer, he/she could not get e.g., a driver's license in many countries.

\section{FOV EXTENSION TO A VR VIEWER}

We extended significantly the FOV of a standard VR viewer. As a base for the primary (front) view, we used a Google Cardboard (Insignia $^{6}$ ) VR viewer and an LG G3 ${ }^{7}$ smartphone, which has a 5.5" screen with a resolution of $1440 \times 2560$ pixels $(515 \mathrm{ppi})$.

For peripheral views we cut out the side areas near the eyepiece of the Cardboard viewer. We added a stack of 3 Fresnel lenses $(58 \times 43$ $\mathrm{mm}, \mathrm{f}=160 \mathrm{~mm}$, groove pitch $0.2 \mathrm{~mm}$, thickness $0.4 \mathrm{~mm}, \mathrm{PVC}$ plastic) and an LG G3 smartphone for each eye. The side lenses and screens were immediately adjacent to the Cardboard lenses at $40^{\circ}$ angles, depicted in Figure 1.

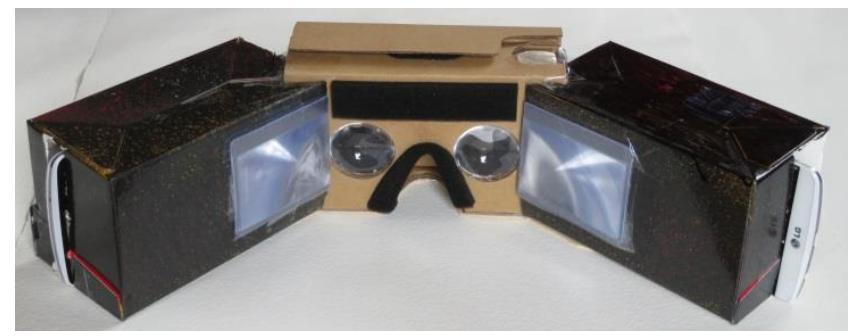

Figure 1. Wide-FOV peripheral extension screens (lenses and smartphones) added to a Google Cardboard.

If lenses of focal lengths $f_{1}, f_{2}$ and $f_{3}$ are thin, the combined focal length $f$ of the stacked lenses is given by the basic lens equation:

$$
\frac{1}{\mathrm{f}}=\frac{1}{\mathrm{f} 1}+\frac{1}{\mathrm{f} 2}+\frac{1}{\mathrm{f} 3}
$$

The optical centers of the Fresnel lenses were $20 \mathrm{~mm}$ from the edge of the side display frame (and from the eyepiece of the main display). The distance from the Fresnel lenses to the screen is $30 \mathrm{~mm}$, and the distance from the lenses to the eye is $20 \mathrm{~mm}$. Fresnel lenses have a black-out effect that occurs if the viewing angle is too acute, but our prototype design avoids such extreme viewing angles.

The extra weight of lens and frame is only tens of grams, and most extra weight (and extra cost of $\sim \$ 100 /$ each) comes from the two side smartphones (149 $\mathrm{g}$ each). Due to magnification of the Fresnel lenses, the side extensions employ less than half of the side screens, as the rest of the screen is beyond human FOV. Also, the peripheral views do not need to be stereoscopic due to properties of human vision. For the side views of this prototype, we used the inner half of the stereoscopic rendering.

No software rectification or image warping according to the optics was used for the peripheral views at this point. Also the magnification of the Cardboard optics and Fresnel lenses did not match exactly, but it was close enough for initial testing.

The visual field has a $5^{\circ}$ gap due to the VR viewer frame between the main and peripheral lenses, but this could be reduced or removed with custom design. Another discontinuity in the visual field is the differences in the display surface normals. However, this did not seem to pose any negative effects for viewing.

\footnotetext{
${ }^{5}$ http://www.wearality.com/wearalitysky/

${ }^{6} \mathrm{http}: / /$ www.bestbuy.com/site/insignia-virtual-realityviewer/5403414.p?skuId=5403414
}

Overall, the FOV is much wider (estimated $200^{\circ}$ horizontal minus the two frame gaps) than with the standard VR viewer $\left(65^{\circ}\right.$ horizontal). Vertical FOV is about $90^{\circ}$.

The image quality (resolution, colors etc.) on the peripheral displays with angled Fresnel lenses is almost as good as in the front view with standard Cardboard viewer. We took photographs from the eye position towards various directions, and the resolution is preserved well in all directions (see Figure 2).
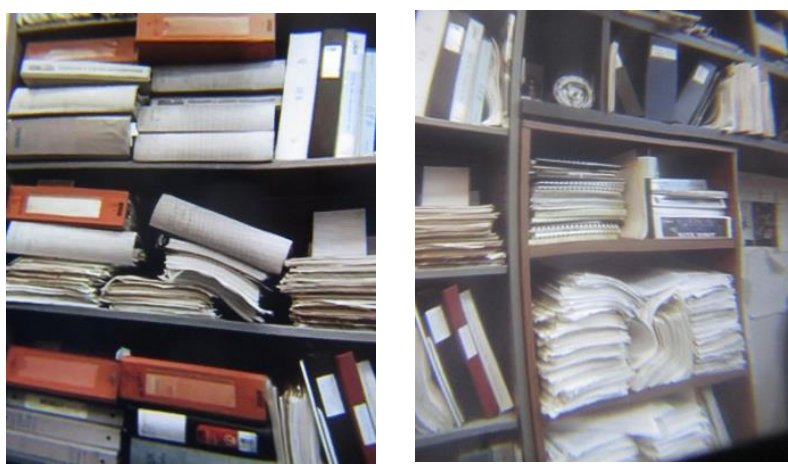

Figure 2. Photographs from the right eye position towards front and $65^{\circ}$ to the side.

The user cannot see precise images in the peripheral areas, but this is more vision-limited than optics-limited. In fact, the screen resolution may not need to be high in the peripheral areas, which also eases requirements on peripheral rendering and optics.

We initially tested the wide FOV with some stereoscopic VR apps, for example a commercial VR app Within (http://with.in/), which is for viewing $360^{\circ}$ spherical videos. It was set for Google Cardboard v2, and we initially adjusted the image on each smartphone correctly by dragging it with finger so that all the three views matched together in static views.

The tracking relied on the internal tracking of each smartphone. As the smartphones are identical, their rotational tracking worked in a coherent way for short periods of time and thus retained synchronized views, even though it (and consequently the views) might get out of sync on longer stretches of use. It worked well for video sections where camera had not moved and thus the scene was not moving. $360^{\circ}$ spherical still images would not need video frame synchronization.

\section{DISCUSSION}

There are several issues and details, which can be improved in our implementation. Proper synchronization between the smartphones or a centralized rendering would be better, even though non-synchronized short-term views were enough for our proof-of-concept prototype. The construction of the prototype was not robust enough for proper user testing. The construction for the side displays could be much smaller and thinner than in the prototype. The resolution of the LG G3 display is higher than required for peripheral vision. Lower-resolution displays on the sides should be adequate and they also lower the cost.

Extending the FOV of current VR viewers usually requires some tear down. The manufacturers could design FOV accessories as attachable, modular side displays, which would require relatively low resolution and processing power. Thin and flexible Fresnel lenses and flexible OLED screens could enable pull-out, pop-up or

${ }^{7}$ http://www.lg.com/us/mobile-phones/g3 
foldable side display flaps for smartphones. Full human FOV is usually not needed, and small side screen stretchers might provide a good compromise and tradeoff for wide FOV in most cases.

Fresnel lenses may enable integrated or slim device structures for casual, immersive VR viewing, along the lines of Rakkolainen et al. [19], Goggle Tech C1-Glass ${ }^{8}$ or Speck PocketVR ${ }^{9}$. When a Fresnel lens is directly touching the screen, it is fully transparent with no optical magnification and little optical artifacts. However, popping up the lens from the screen, or possibly a dual-screen clamshell smartphone could enable compact, embedded and wide-FOV VR viewers.

The perceptual issues need to be taken into account and user testing is needed. The various psychophysical effects of super-wide FOV provide interesting research opportunities.

\section{CONCLUSIONS}

We have significantly extended the FOV of a standard VR viewer. The proof-of-concept prototype has super-wide FOV with high resolution. The peripheral side lenses and screens produced almost as good images as the front display, which is very promising, considering the low price and bulk quality of the Fresnel lenses, and properties of the human eye and its peripheral vision.

The extended FOV was more immersive and natural than the limited standard FOV of a Cardboard viewer. Super-wide FOV seems to enhance the user experience significantly according to our initial and informal testing. Our extended VR headset suggests that it is an intriguing and promising option for VR viewers. It can be made fairly low-cost, light-weight and compact. This may have interesting implications for $3 \mathrm{D}$ user interfaces, $\mathrm{VR}, 360^{\circ}$ video and other applications. We will continue to develop more robust hardware and software to test these issues further with better prototypes and larger user populations.

\section{ACKNOWLEDGMENTS}

We thank all the colleagues and reviewers who provided helpful comments on this paper. This work is partially funded by the Finnish Funding Agency for Innovation (Tekes, \#1444/31/2016), Alfred Kordelin Foundation and NSF grant \#1423676.

\section{REFERENCES}

[1] Ardouin, J., Lécuyer, A., Marchal, M., Riant, C. and Marchand, E. 2012. FlyVIZ: a novel display device to provide humans with $360^{\circ}$ vision by coupling catadioptric camera with hmd. In Proceedings of the 18th ACM symposium on Virtual reality software and technology (VRST'2012). ACM, 41-44.

[2] Arthur, K. 2000. Effects of Field of View on Performance with Head-Mounted Displays. Doctoral thesis. Univ. of North Carolina at Chapel Hill.

[3] Baek, J., Jung, J. and Kim, G. 2005. Head mounted display with peripheral vision. In Proceedings of the 2005 International Conference on Augmented Tele-existence. ACM, 282.

[4] Brown, L. and Boger, Y. 2008. Applications of the Sensics Panoramic HMD. In Proceedings of the SID Symposium Digest of Technical Papers 39, 1. SID, 77-80.

[5] Bungert, C. 2017. HMD/headset/VR-helmet comparison chart. http://www.stereo3d.com/hmd.htm. Accessed August 2, 2017.

[6] Cakmakci, O. and Rolland, J. 2006. Head-worn displays: a review. J. Display Technology 2, 3. IEEE, 199-216.
[7] Cheng, D., Wang Y., Hua H. and Sasian, J. 2011. Design of a wide-angle, lightweight head-mounted display using freeform optics tiling. Opt. Lett. 36, 11. OSA, 2098-100.

[8] Chinnok, C. 2017. Understanding Trade-offs in Microdisplay and Direct-View VR Headset Designs, Insightmedia report, February 2017, www.insightmedia.info.

[9] Fan, K., Huber, J., Nanayakkara, S. and Inami, M. 2014. SpiderVision: extending the human field of view for augmented awareness. In Proceedings of the 5th Augmented Human Int. Conf. (AH'14). ACM, Article 49.

[10] Howlett, E. 1990. Wide angle orthostereo. In Proceedings of the 1990 SPIE Conference on Stereoscopic Displays and Applications. SPIE, 210-223.

[11] Jay, C. and Hubbold, R. 2003. Amplifying Head Movements with Head-Mounted Displays, Presence, 12, 3, 268-276.

[12] Jones, J., Swan, J. and Bolas, M. 2013. Peripheral Stimulation and its Effect on Perceived Spatial Scale in Virtual Environments. IEEE Trans. on Visualization \& Computer Graphics, 19, 4 (March 2013), 701-710.

[13] Kiyokawa, K. 2007. A wide field-of-view head mounted projective display using hyperbolic half-silvered mirrors. In Proceedings of the ISMAR'2007 Conference. IEEE, 1-4.

[14] Kress, B. and Starner, T. 2013. A review of head-mounted displays (HMD) technologies and applications for consumer electronics. In Proceedings of the SPIE 8720, Defense, Security, and Sensing. SPIE.

[15] Lin, J., Duh, H., Parker, D., Abi-Rached, H. and Furness, T. 2002. Effects of Field of View on Presence, Enjoyment, Memory and Simulator Sickness in a Virtual Environment, In Proc. of the IEEE Virtual Reality 2002. IEEE, 164-171.

[16] Orlosky, J., Wu, Q., Kiyokawa, K., Takemura, H. and Nitschke, C. 2014. Fisheye vision: peripheral spatial compression for improved field of view in head mounted displays. In Proceedings of the 2nd ACM symposium on Spatial user interaction (SUI'2014). ACM, 54-61.

[17] Patterson, R., Winterbottom, M. and Pierce, B. 2006. Perceptual Issues in the Use of Head-Mounted Visual Displays. Human Factors 48, 3. Human Factors \& Ergon. Soc., 555-573.

[18] Pelz, J. and Canosa, R. 2001. Oculomotor behavior and perceptual strategies in complex tasks. Vision Research, 41(25), 3587-3596.

[19] Rakkolainen, I., Raisamo, R., Turk, M., Höllerer, T. and Palovuori, K. 2016. Casual Immersive Viewing with Smartphones. In Proceedings of the 20th International Academic Mindtrek Conference 2016.

[20] Rakkolainen, I., Turk, M. and Höllerer, T. 2016. A Superwide-FOV Optical Design for Head-Mounted Displays. In Proceedings of the Joint 26th Int. Conf. on Artificial Reality and Telexistence \& the 21th Eurographics Symp. on Virtual Environments (ICAT-EGVE 2016). Eurographics, 45-48.

[21] Rash, C., Russo, M., Letowski, T. and Schmeisser, E. (Ed.). 2009. Helmet-Mounted Displays: Sensation, Perception and Cognition Issues. U.S. Army Aeromedical Research Lab.

[22] Ren, D., Goldschwendt, T., Chang, Y. and Höllerer, T. 2016. Evaluating Wide-Field-of-View Augmented Reality with

\footnotetext{
${ }^{9}$ http://www.speckproducts.com/pocket-vr.html
}

\footnotetext{
${ }^{8} \mathrm{http}: / / g o g g l e t e c h . n e t /$
} 
Mixed Reality Simulation. In Proceedings of the IEEE VR 2016. IEEE, 93-102.

[23] Sutherland, I. 1968. A Head-mounted Three-dimensional Display. In Proceedings of the AFIPS Fall Joint Computer Conference 1968, Thompson Books, Vol. 3, 757-764.
[24] Xiao, R. and Benko, H. 2016. Augmenting the Field-of-View of Head-Mounted Displays with Sparse Peripheral Displays. In Proc. of the ACM CHI 2016 Conference. 1221-1232. 